\title{
Sparse Scale-Space Decomposition of Volume Changes in Deformations Fields
}

\author{
Lorenzi Marco ${ }^{1}$, Bjoern H Menze ${ }^{1,2}$, Marc Niethammer ${ }^{3}$, Nicholas Ayache ${ }^{1}$, \\ and Xavier Pennec ${ }^{1}$ for the Alzheimer's Disease Neuroimaging Initiative* \\ 1 Project Team Asclepios, INRIA Sophia Antipolis, France ${ }^{\star \star}$ \\ 2 Computer Vision Laboratory, ETH Zurich, Switzerland \\ 3 Department of Computer Science, UNC Chapel Hill, US
}

\begin{abstract}
Anatomical changes like brain atrophy or growth are usually not homogeneous in space and across spatial scales, since they map differently depending on the anatomical structures. Thus, the accurate analysis of volume changes from medical images requires to reliably localize and distinguish the spatial changes occurring at different scales, from voxel to regional level. We propose here a framework for the sparse probabilistic scale-space analysis of volume changes encoded by deformations. Our framework is based on the Helmoltz decomposition of vector fields. By scale-space analysis of the scalar pressure map associated to the irrotational component of the deformation, we robustly identify the areas of maximal volume changes, and we define a consistent sparse decomposition of the irrotational component. We show the effectiveness of our framework in the challenging problem of detecting the progression of tumor growth, and in the group-wise analysis of the longitudinal atrophy in Alzheimer's disease.
\end{abstract}

\section{Introduction}

Modeling the structural changes of organs and tissues is an important goal of medical image analysis. Anatomical changes like brain atrophy or growth are usually not homogeneous in space and across spatial scales, since they differ for different anatomical structures. For instance, Alzheimer's disease leads to cortical thinning, which is local and detectable at millimiter scale on the gray matter ribbon, as well as regional or global matter loss, which involves entire anatomical regions like hippocampi or brain ventricles. Thus, the accurate analysis of anatomical changes requires to reliably localize and distinguish the spatial support of changes occurring at different scales, from voxel to regional level.

* Data used in preparation of this article were obtained from the Alzheimer's Disease Neuroimaging Initiative (ADNI) database (www.loni.ucla.edu/ADNI). As such, the investigators within the ADNI contributed to the implementation of ADNI and/or provided data but did not participate in analysis or writing of this report.

** This work was partially funded by the European Research Council (ERC advanced Grant MedYMA) 
Scale-space analysis of image data is a classical topic in computer vision, and different applications have been proposed in the field of medical imaging. The main issue when working with medical images concerns the statistical assessment of spatial areas of meaningful signal. For instance, in [10] Gaussian field theory was used to define a sparse set of statistically significant regions of activation across spatial scales in PET images.

Anatomical changes can be detected by non-linear registration, which models the differences between pairs of images as dense deformation fields in the image space. Analysis of dense deformation fields is usually challenging due to the high dimensionality of the data. For this reason, methods have been proposed for estimating deformations by considering different spatial scales [7], by enforcing spatial sparsity [1], or by combining the approaches to provide sparse scale-space registration [8]. Even though these approaches provide a richer description of the deformations, the interpretation of the results is still challenging. In fact, registration does not provide a statistical measure for assessing the significant locations of the estimated anatomical changes, and might thus lack robustness.

A different approach for the study of deformations consists in analyzing the encoded spatial changes posterior to the registration, for instance by voxel-byvoxel statistical analysis of associated Jacobian determinant. Jacobian analysis is however local, not very robust, and prone to multiple comparison problems.

Helmoltz decomposition parameterizes a given deformation as the sum of irrotational and divergence-free components [2]. The irrotational component is a dense vector field which encodes the local volume changes associated to the deformation, and therefore which completely describes the estimated atrophy and growth processes. The irrotational component is the gradient of a scalar apparent pressure map, whose topological analysis enables the identification of areas of local volume changes. This idea was used in [4] to localize the areas of relevant brain atrophy in Alzheimer's disease (AD) from a set of extremal pressure points. In this case the extremal points were manually selected according to prior knowledge of the typical AD atrophy pattern. This is normally not possible when studying other biological processes, like the evolution of brain tumors, where regions of potential volume change are usually unknown. Moreover, the approach proposed in [4] is not very robust, since the identification of local extremal pressure points is very sensitive to the image noise, and critically depends on the considered spatial scale.

\subsection{Contributions and paper's structure}

In this work we propose a fully automatic probabilistic framework for the sparse scale-space analysis of the volume changes encoded by deformations.

Since we focus on the study of volume changes, our framework is based on the irrotational field obtained by the Helmoltz decomposition. The irrotational field is the gradient of a scalar pressure field. Our contributions are as follows:

- Scale-space analysis of extremal pressure point. We introduce a probabilistic framework for the scale-space identification of local extremal pressure points. 
These points are the local sources and sinks of the volume changes associated to the deformation. This contribution provides the robust identification of locations of maximal volume change, and is detailed in Section 3.1.

- Critical Regions Associated to Probabilistic Extremal Pressure Points. Given the probabilistic extremal points we identify the areas of maximal volume changes (critical regions) associated to the deformation. This step, detailed in Section 3.2, enables to robustly estimate the critical regions consistently with respect to the irrotational component.

- Sparse Description of the Velocity from the Critical Regions. We use the critical areas to finally decompose the irrotational field in a sparse set of components (Section 3.3). This last contribution enables a fully consistent and simple sparse decomposition of the volume changes encoded by the deformation.

- Application: modeling tumor evolution and group-wise atrophy in Alzheimer's disease. In Section 4 we prove the effectiveness of our framework in the challenging problem of detecting and localizing progressing tumor growth in the brain, and in the group-wise modeling of the longitudinal atrophy in Alzheimer's disease.

\section{Topology of Pressure Fields and Volume Changes in Deformations}

Our framework is based on non-linear transformations parameterized by stationary velocity fields, provided for instance by the LCC-logDemons registration algorithm [5]. Consider a deformation $\exp (\mathbf{u})$. Following [4] we can decompose the stationary velocity field $\mathbf{u}$ according to the Helmoltz decomposition as the sum of an irrotational and of a divergence-free component $\mathbf{u}=\nabla P+\nabla \times A$. The divergence-free component describes the rotational part of the velocity which is not associated to volume changes. The irrotational velocity field $\mathbf{v}=\nabla P$ is the gradient of an apparent scalar pressure field $P$, which encodes the volume changes associated to $\mathbf{u}$. In fact, local volume changes are associated to local variations of the pressure $P$.

Scale-Space Analysis of Extremal Pressure Points Critical points of local maxima and minima of the pressure field are respectively local attractors and generators of the irrotational velocity field, and therefore characterize area of contraction and expansion. Each local extremum defines an area of maximal volume change, called critical region, which is a peak or a basin for the pressure field, and which is characterized by respectively maximum inwards or outwards flux of the irrotational field across its boundaries (Figure 1). From the divergence theorem we know that the integral over basins and peaks of the divergence of the irrotational velocity field is respectively negative or positive. 
The spatial location of critical regions is related to the local pressure variation. Critical regions can be contained in larger zones of pressure extrema. In fact, the global irrotational field is the contribution of several components acting differently across scales, and which are associated to critical regions mapping to different spatial locations. It is therefore of interest to analyze the topology of pressure fields at different spatial scales in order to disentangle and separately analyze its different components. In the following section we propose a robust framework for the reliable scale-space identification of critical regions associated to irrotational fields of anatomical deformations.

\section{Scale-Space Identification of Critical Areas}

Let $\mathbf{v}=\nabla P$ be an irrotational velocity field, and let the pressure $P$ be zero outside the image boundaries. We consider a set of increasing local Gaussian neighborhoods $\mathcal{G}_{\sigma_{k}}$. Let $\mathcal{G}_{0} * P=P$ and $\lim _{n \rightarrow \infty} \mathcal{G}_{\sigma_{k}} * P=0$, and consider the following partition of $P$ through convolutions $P=\sum_{k}\left(\mathcal{G}_{\sigma_{k}}-\mathcal{G}_{\sigma_{k+1}}\right) * P=\sum_{k} P_{k}$. The $P_{k}$ obtained by the difference of Gaussians (DoG) operator are the bandpass filtering of $P$ at scale $\sigma_{k}-\sigma_{k+1}$. DoG is used in classical scale-space analysis as enhancement operator for blob detection, which are defined by identifying the maximal points in scale-space $q=\operatorname{argmax}_{(k, x)}\left(P_{k}(x)\right)[6]$.

In the present study we want to robustly detect the scale-space extremal points (maxima and minima) in the presence of potentially noisy data. For this purpose we propose a probabilistic framework for assessing the uncertainty of the position of such local extrema.

\subsection{Probabilistic Scale-Space Detection of Extremal Pressure Points}

We define here a probabilistic framework for the reliable definition of scalespace extremal pressure points. Let $\left(P_{n}(x)\right)$ be the $4 \mathrm{D}$ scale-space associated to a pressure field $P$ at the set of scales $\sigma=\left\{\sigma_{k}-\sigma_{k+1}\right\}$. Let $C_{k}$ be the probabilistic region at the scale level $k$ for the location of the maximal point $\mu_{k}$. We set:

$$
\mathcal{P}\left(C_{k} \mid \sigma\right)=\mathcal{P}\left(C_{k} \mid \mu_{k}\right) \mathcal{P}\left(\mu_{k} \mid \sigma\right)
$$


This way, $C_{k}$ depends on the proximity to the maximal point $\mu_{k} \mathcal{P}\left(C_{k} \mid \mu_{k}\right)$, conditioned by the probability of $\mu_{k}$ to be maximal across scales $\mathcal{P}\left(\mu_{k} \mid \sigma\right)$. We compute $\mathcal{P}\left(\mu_{k} \mid \sigma\right)$ as the cumulative probability of the value of $\mu_{k}$ in a $4 \mathrm{D}$ neighborhood of $\left(P_{n}(x)\right)$ centered in $\mu_{k}$. The term $\mathcal{P}\left(C_{k} \mid \mu_{k}\right)$ is instead computed from the Bayes formula as

$$
\mathcal{P}\left(C_{k} \mid \mu_{k}\right) \approx \mathcal{P}\left(\mu_{k} \mid C_{k}\right) \mathcal{P}\left(C_{k}\right),
$$

where $\mathcal{P}\left(C_{k}\right)$ is assumed uniform and constant across scales, and $\mathcal{P}\left(\mu_{k} \mid C_{k}\right)$ is the probability of $\mu_{k}$ to be maximum given the neighborhood $C_{k}$. We express it as:

$$
\mathcal{P}\left(\mu_{k} \mid C_{k}\right)=\frac{\sum_{x} \frac{1}{2}\left(1+<\nabla P_{k}(x), d(x)>\right) \exp \left(-\frac{\|d(x)\|^{2}}{2\left(\sigma_{k}^{2}\right)}\right)}{\sum_{x} \exp \left(-\frac{\|d(x)\|^{2}}{2\left(\sigma_{k}^{2}\right)}\right)},
$$

with $d(x)=\mu_{k}-x$, and $\langle a, b\rangle=\frac{a}{\|a\|^{2}} \frac{b}{\|b\|^{2}}$. Equation (1) states that the region $C_{k}$ is composed of points close to maximal points $\mu_{k}$, and oriented along the flow lines of $P_{k}$ generated by $\mu_{k}$.

\subsection{Critical Regions Associated to Probabilistic Extremal Pressure Points}

Now that we have robustly identified the significant locations of extremal pressure points across scales, we are interested in detecting the critical regions generated by such points. In particular, since extremal points are sources (resp. sinks) of the irrotational field, the critical regions are pressure basins (resp. peaks) characterized by maximum flux of the irrotational field across their boundaries.

Let consider the area $C_{k}$ such that $\mathcal{P}\left(C_{k} \mid \sigma\right)>0.6$, i.e. the significant area at the scale $k$ for the critical point $\mu_{k}$. Let $\mathbf{v}_{k}=\nabla P_{k}$ be the irrotational field associated to the scale level $k^{4}$, and let $M^{\mu_{k}}$ be the region containing $C_{k}$ for which the flux of $\mathbf{v}_{k}$ is maximum. In [9] it was shown that $M^{\mu_{k}}$ is the limit of the evolution of the surface $\partial C_{k}$ according to $\frac{d\left(\partial C_{k}\right)}{d t}=\left(\nabla \cdot \mathbf{v}_{k}\right) \cdot \bar{n}$, where $\bar{n}$ is the normal to $\partial C_{k}$. The region $M^{\mu_{k}}$ can be therefore obtained from $C_{k}$ by iterating the above formula to convergence through a classical gradient descent approach.

\subsection{Sparse Description of the Velocity from the Critical Regions}

Given a scale level $k$, the critical regions $M^{\mu_{k}^{i}}$ are the significant spatial support of the spatially dense component $\mathbf{v}_{k}$. The set of regions $M=\cup_{i, k} M^{\mu_{k}^{i}}$ represents the loci of significant volume changes of the deformation in scale-space, thus the significant sparse spatial support of the irrotational field $\mathbf{v}=\sum_{k} \mathbf{v}_{k}$.

The set $M$ therefore decomposes the irrotational velocity in space 1) robustly through the probabilistic scale-space analysis of critical regions, and 2) consistently with its flow properties, in order to preserve only the most representative volume changes.

\footnotetext{
${ }^{4}$ We note that $\mathbf{v}=\nabla P=\nabla\left(\sum_{k} P_{k}\right)=\sum_{k} \nabla P_{k}=\sum_{k} \mathbf{v}_{k}$
} 

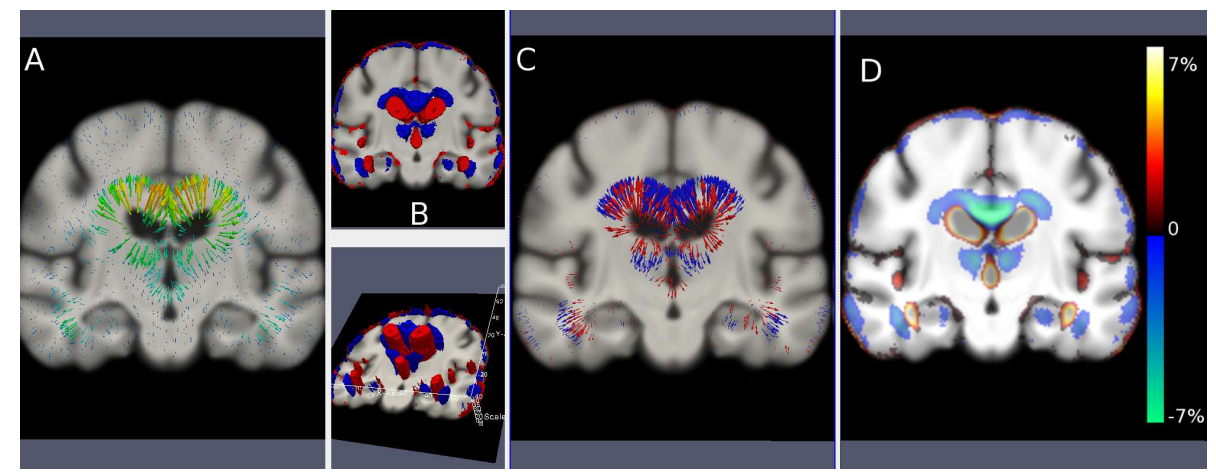

Fig. 2: Group-wise scale-space analysis for the 1-year brain atrophy in $30 \mathrm{AD}$ patients. A) Group-wise irrotational field B) Group-wise scale-space critical regions (red: expansion, blue: contraction). The Z-axis represents the scale of detection of critical regions (finer to coarser). The most persistent atrophy (taller blobs) is localized in the ventricles and in the hippocampi. Cortical atrophy is instead detectable at smaller scales. C) C) Decomposition of the irrotational field in expanding (red), and contracting (blue) components. The decomposition is obtained by masking the velocity field in A) by the areas of B). D) Cumulative percentage volume changes across scales associated to the critical regions.

\section{Application}

We provide two very different applications of the proposed framework. First, we apply it to the modeling of the group-wise longitudinal atrophy in Alzheimer's disease (AD). The aim is to localize the key anatomical regions of AD atrophy at different scales, from local (cortical thinning) to regional level, for instance for the hippocampal atrophy. Second, we use our framework for detecting and localizing tumor growth that occurs after the patient has been treated. In this case, scalespace analysis enables to separate those changes which are more persistent and directly related to the tumor process, from those more subtle ones which are indirectly related to the tumor or induced by the treatment, like local atrophy or swelling of ventricles/sulci.

The following results were obtained by considering $\sigma_{0}=0 \mathrm{~mm}$, and $\sigma_{k}=$ $\sigma_{k-1}+0.5 m m, k=1, \ldots, 16$.

Modeling Group-Wise Atrophy in AD. We selected 30 patients affected by AD from the ADNI dataset. For each subject the baseline brain 3D T1 structural image was registered to the one-year follow-up with the log-Demons algorithm. Subject specific irrotational fields were transported in a common reference space by using a method proposed elsewhere [3], and the corresponding pressure components were analyzed with the proposed framework.

Figure $2 \mathrm{~A}$ shows the group-wise average of the transported irrotational fields. In Figure 2B we show the scale-space group-wise average critical areas obtained 


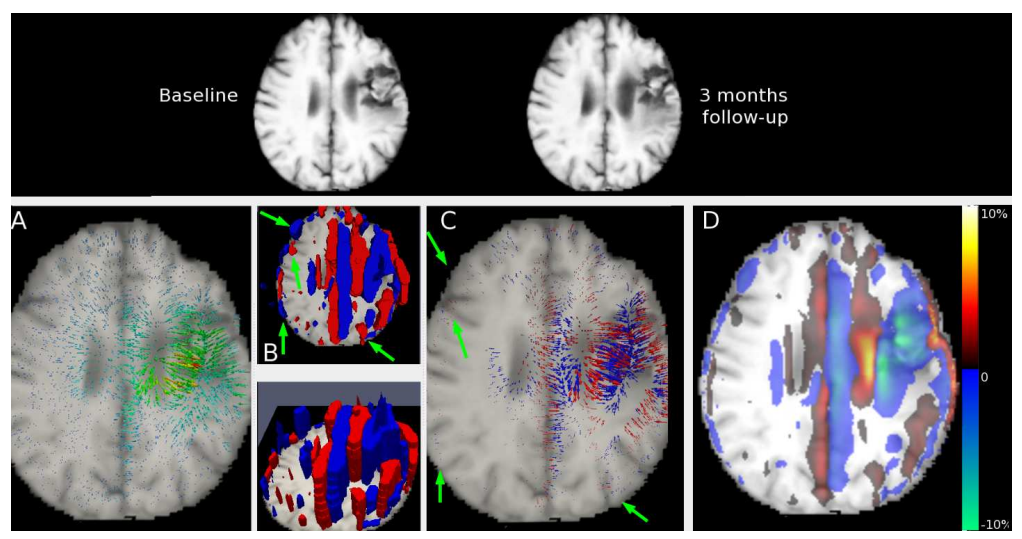

Fig. 3: Scale-space analysis for longitudinal brain tumor evolution. Top: 3 months follow-up time series. Bottom: A) Irrotational field estimated by the longitudinal registration. B) Scale-space analysis of the critical areas associated to the irrotational field (blue: contraction, red: expansion). Persistent volume changes are visible in the tumor core and in the ventricles (taller blobs). We capture the shift of the midline axes towards the right hemisphere, and we find also very localized atrophy at the brain boundaries/sulci (green arrows). C) Decomposition of the irrotational field in expanding (red), and contracting (blue) components. The decomposition is obtained by masking the velocity field in A) by the areas of B). D) Cumulative percentage volume changes across scales associated to the critical regions.

with our framework. We note that the most persistent atrophy is localized in the ventricles and in the hippocampi. Cortical atrophy is instead detectable at smaller scales. When masking the average irrotational component by this sparse set of regions (Figure 2C) we preserve the significant atrophy pattern while discarding the more local and less relevant deformation patterns.

Modeling Brain Tumor Evolution. We chose 3D T1 MRI from a patient treated with Avastin. The time difference between the images is 3 months. Avastin suppresses characteristic intensity changes in T1 and T2 MRI, which limits the usefulness of traditional change detection methods that rely on the detection of advancing edema or tumor core boundaries.

Scale space analysis of the tumor evolution is shown in Figure 3, indicating and localizing significant volume changes. We find more persistent volume changes corresponding to shrinkage in the tumor core area (blue), and to decompression of tissue surrounding the tumor following the successful therapy (red) (Figure 3B). Moreover, the present analysis is able to capture the shift of the midline axes towards the right hemisphere, which is detectable at smaller scales, as well as the local atrophy at the brain boundaries/sulci (green arrows). In the given case we do not find indications of recurring growth, but only indications of "stable disease". 


\section{Conclusions and Perspectives}

Scale-space analysis of the irrotational velocity field provides a new and interesting instrument for the analysis of atrophy processes, for instance to distinguish critical regions which are characteristic of a given scale from those which remain persistent across scales. Our experimental results showed how this framework can be already successfully applied to clinical problems for robustly differentiate volume changes across spatial scales. The proposed robust analysis of critical regions defines the relevant areas of action of the irrotational velocity, and provide thus a topologically consistent and sparse description of the deformation.

The present study is a step towards the consistent topological analysis of deformations. For example, one can define a tree-like structure of the pressure field, where each level of the tree is a given spatial scale, and the branches connect critical regions which are nested across scales. This way, topology of deformations can be studied with Morse-Smale or graph theory.

Finally, the presented work can be extended to the analysis of sequences of deformations, for instance for detecting anatomical changes in time series of several images. For this purpose, the consistent extension of the presented framework to 5 -dimensional data (space+scale+time) is required.

\section{References}

1. Durrleman, S., Allassonnière, S., Joshi, S.C.: Sparse adaptive parameterization of variability in image ensembles. International Journal of Computer Vision 101(1), 161-183 (2013)

2. Hansen, M.S., Larsen, R., Christensen, N.V.: Curl-gradient image warping - introducing deformation potentials for medical image registration using Helmholtz decomposition. In: VISAPP 2009. vol. 1, pp. 179-185 (2009)

3. Lorenzi, M., Ayache, N., Pennec, X.: Schild's Ladder for the parallel transport of deformations in time series of images. In: Information Processing in Medical Imaging - IPMI. vol. 22, pp. 463-474 (2011)

4. Lorenzi, M., Ayache, N., Pennec, X.: Regional flux analysis of longitudinal atrophy in Alzheimer's disease. In: Medical Image Computing and Computer-Assisted Intervention - MICCAI. LNCS, Springer (Oct 2012)

5. Lorenzi, M., Frisoni, G.B., Ayache, N., Pennec, X.: LCC-Demons: A robust and accurate diffeomorphic registration algorithm. NeuroImage (2013), to appear

6. Lowe, D.G.: Object recognition from local scale-invariant features. In: Proceedings of ICCV. vol. 2, pp. 1150-1157 (1999)

7. Seiler, C., Pennec, X., Reyes, M.: Geometry-Aware Multiscale Image Registration Via OBBTree-Based Polyaffine Log-Demons. In: Medical Image Computing and Computer-Assisted Intervention - MICCAI. vol. 14, pp. 631-638 (2011)

8. Sommer, S., Lauze, F., Nielsen, M., Pennec, X.: Sparse multi-scale diffeomorphic registration: the kernel bundle framework. J. of Mathematical Imaging and Vision (2012), in press

9. Vasilevskiy, A., Siddiqi, K.: Flux maximizing geometric flows. IEEE Trans. Pattern Anal. Mach. Intell. 24(12), 1565-1578 (Dec 2002)

10. Worsley, K.J., Marrett, S., Neelin, P., Evans, A.C.: Searching scale space for activation in PET images. Human Brain Mapping 4, 74-90 (1996) 